\title{
Normal growth and development in mice over-expressing the CCN family member WISP3
}

\author{
Yukio Nakamura • Yajun Cui • Carol Fernando • \\ Wendy E. Kutz • Matthew L. Warman
}

Received: 12 January 2009 /Accepted: 25 February 2009/Published online: 29 April 2009

(C) The Author(s) 2009. This article is published with open access at Springerlink.com

\begin{abstract}
Loss-of-function mutations in the gene WISP3 cause the autosomal recessive human skeletal disease Progressive Pseudorheumatoid Dysplasia, whereas mice with knockout mutations of Wisp3 have no phenotype. The lack of a phenotype in the Wisp 3 knockout mice has constrained studies of the protein's in vivo function. Overexpression experiments in zebrafish indicated that WISP3 may function as a BMP and Wnt signaling modulator. To determine whether these biologic activities are retained in mice, we created two strains of transgenic mice that overexpress WISP3 in a broad array of tissues. Despite strong and persistent protein over-expression, the transgenic mice remained phenotypically indistinguishable from their nontransgenic littermates. Surprisingly, WISP3 contained in conditioned medium recovered from transgenic mouse primary kidney cell cultures was able to bind BMP and to inhibit BMP signaling in vitro. Factors that account for the difference between the in vitro and in vivo activities of WISP3 remain unknown. At present, the mouse remains a challenging model organism in which to explore the biologic function of WISP3.
\end{abstract}

Y. Nakamura $\cdot$ M. L. Warman

Howard Hughes Medical Institute, Orthopaedic Research Laboratories, Department of Orthopaedic Surgery and Genetics, Children's Hospital and Harvard Medical School,

Boston, MA, USA

Y. Cui $\cdot$ C. Fernando $\cdot$ W. E. Kutz

Department of Genetics, Case Western Reserve University,

Cleveland, $\mathrm{OH}$, USA

Y. Nakamura $(\bowtie)$

Howard Hughes Medical Institute, Orthopaedic Research

Laboratories, Children's Hospital and Harvard Medical School,

Boston, MA 02115, USA

e-mail: yukio.nakamura@childrens.harvard.edu

e-mail: yxn14@ims.u-tokyo.ac.jp
Summary of article. Transgenic mice that broadly overexpress WISP3 were created to search for in vivo biologic activities, since mice that lack WISP3 were normal. Surprisingly, transgenic mice were also phenotypically indistinguishable from wild-type animals. The mouse is a challenging model organism in which to explore the biologic function of WISP3.

Keywords WISP3 $\cdot \mathrm{CCN} \cdot \mathrm{BMP} \cdot \mathrm{Wnt}$

\section{Introduction}

Mutations in the secreted protein Wnt-1 inducible signaling pathway protein 3 (WISP3) cause the autosomal recessive, human, skeletal disease, Progressive Pseudorheumatoid Dysplasia (PPD) (Hurvitz et al. 1999). Individuals with PPD appear normal at birth, develop clinical symptoms between 3 and 8 years-of-age, and progress to severe degenerative joint disease requiring joint replacement surgery by their second decade of life (Wynne-Davies et al. 1982; Spranger et al. 1983; el-Shanti et al. 1997; Sewairi and Warman 2003). Individuals with PPD have no organ involvement outside of their skeletal system. Consequently, in humans, the principal role of WISP3 appears to involve skeletal growth and cartilage homeostasis.

Given the cartilage phenotype in patients with PPD, in vitro studies using primary human chondrocytes or chondrocyte cell lines have been performed to precisely define the biologic function of WISP3. Over-expression of human WISP3 (hWISP3) in human chondrocyte cell lines was reported to increase expression of type II collagen, aggrecan, and Sox9, as determined by semi-quantitative reverse transcription polymerase chain reaction (RT-PCR) and/or by Western blot (Sen et al. 2004). Human primary chondrocytes recovered 
from a single patient with PPD at the time of joint replacement surgery were noted to have increased cell proliferation and abnormal matrix metalloproteinase processing when compared to wild-type chondrocytes from a single control (Zhou et al. 2007). However, the in vivo relevance for either of these findings has not been determined. The ease of manipulating WISP3 expression in zebrafish by injecting morpholinos and mRNA into fertilized eggs allowed us to employ knockdown and over-expression approaches to explore in vivo actions of zebrafish WISP3 (zWISP3) (Nakamura et al. 2007). These studies indicated that zWISP3 was capable of modulating canonical BMP and Wnt signaling in developing zebrafish (Nakamura et al. 2007).

WISP3 is a member of the $\mathrm{CCN}$ protein family. Mammalian CCN family members have been implicated in a broad array of biologic processes (Chen and Lau 2008) and are characterized by shared conserved structural domains (Rachfal and Brigstock 2005; Leask and Abraham 2006). One of these domains, a carboxy-terminal cystine knot, is also found in several known BMP and Wnt signaling modulators such as Noggin, SOST, and WISE, as well as in members of the TGF- $\beta$ superfamily (Piccolo et al. 1999; Brunkow et al. 2001; Brigstock 2003; Itasaki et al. 2003; Avsian-Kretchmer and Hsueh 2004; Perbal 2004). Consequently, the function of WISP3 in mammalian cartilage could be to fine tune BMP and Wnt signaling. Complicating the in vivo study of WISP3 function in humans and mice is that, in our hands, it's expression is detectable only by RTPCR, and not by in situ hybridization or immunohistochemistry (Kutz et al. 2005). Furthermore, a targeted knockout mutation in mouse Wisp3 (mWisp3) caused no detectable phenotype (Kutz et al. 2005). One potential explanation for this finding is functional redundancy in mice between WISP3 and other CCN family members or BMP and Wnt regulators. Therefore, we created transgenic mice that broadly over-express WISP3 to determine whether an in vivo biologic activity could be identified.

Here we report that WISP3 over-expressing mice are viable, fertile, and phenotypically indistinguishable from their non-transgenic littermates. This result is surprising since the WISP3 protein expressed by primary kidney cell cultures from the transgenic mice retained the ability to bind $\mathrm{BMP}$ and to inhibit BMP signaling in vitro. We conclude that delineating the in vivo roles of WISP3 in mammals may not be possible unless factors responsible for differences in the protein's in vivo and in vitro activity are identified.

\section{Materials and methods}

Generation of mWisp3 cDNA Full-length mWisp3 cDNA (Genbank accession number: XP_282903) was amplified by RT-PCR using mouse testis cDNA and cloned into the Not $I$ and Xba $I$ sites of pcDNA3.1 vector (Invitrogen). Constructs were sequenced to verify that no PCR-induced mutations had occurred and there was no difference between the mWisp3 clone and the Genbank sequence.

Transient transfection of HEK293T cells with the mWisp3 expression vectors used to produce transgenic mice HEK293T cells were grown in Dulbecco's Minimum Essential Medium (DMEM) (Invitrogen) supplemented with $10 \%$ fetal bovine serum (FBS) and 1\% penicillin/ streptomycin (PSM) (Invitrogen). Cells were maintained in $10 \mathrm{~cm}$ tissue culture plates (Falcon) at $37^{\circ} \mathrm{C}$ with $5 \% \mathrm{CO}_{2}$ in a humidified incubator. HEK293T cells were transfected at $70-80 \%$ confluency with $4 \mu \mathrm{g}$ of empty expression vector or vector containing mWisp3 cDNA using LipofectAmine/Plus Reagent (Invitrogen) in $6.5 \mathrm{~mL}$ serum-free DMEM. Four hours (h) after transfection, $6.5 \mathrm{~mL}$ of DMEM supplemented with $20 \%$ FBS was added to the cultures and allowed to incubate overnight. The medium was then replaced with $10 \mathrm{~mL}$ of DMEM containing $10 \%$ FBS for $24 \mathrm{~h}$ followed by $5 \mathrm{~mL}$ of serum-free DMEM for $24 \mathrm{~h}$. Conditioned medium was then recovered and centrifuged at $16,000 \mathrm{~g}$ for $5 \mathrm{~min}$. One-hundred-twentyfive $\mu \mathrm{L}$ of supernatant was then acetone precipitated $(5: 1$ acetone:medium) overnight at $-20^{\circ} \mathrm{C}$ followed by centrifugation at $16,000 \mathrm{~g}$ for $5 \mathrm{~min}$. Acetone was poured off and the pellets were dried before re-suspension in $40 \mu \mathrm{L} 1 \times$ sodium dodecyl sulfate (SDS) sample loading buffer at $55^{\circ} \mathrm{C}$ for $10 \mathrm{~min}$. Cell lysate was recovered by scraping following incubation with $1 \mathrm{~mL}$ RIPA buffer $(150 \mathrm{mM} \mathrm{NaCl}, 0.5 \%$ deoxycholate, $1 \%$ NP-40, $0.1 \% \mathrm{SDS}$, and $50 \mathrm{mM}$ Tris, $\mathrm{pH} 8.0$ ) for $5 \mathrm{~min}$. Cell lysate was centrifuged at $16,000 \mathrm{~g}$ for 5 min to clear insoluble cellular debris. Equal volumes of supernatant and $2 \times$ SDS sample loading solution were then mixed. $\beta$-mercaptoethanol was added to cell lysate and conditioned medium samples $(5 \% \mathrm{v} / \mathrm{v})$ prior to boiling and separation by SDS-PAGE.

Generation of mWisp3 transgenic mice The plasmid vector pcDNA3.1, which drives transgene expression in multiple tissues via a CMV promoter (Yu et al. 2005; Valverde et al. 2008), was used to produce one transgenic strain of mice (CMV-WISP3). The pCAGGS vector, which drives transgene expression using a chicken- $\beta$-actin promoter/CMV enhancer (Niwa et al. 1991), was used to produce another transgenic strain ( $\beta$-actin-WISP3). Full-length $m$ Wisp3 cDNA was subcloned into the EcoRI site of pCAGGS vector. Plasmid DNA was injected into the pronuclei of B6-SJL/F2 oocytes and placed into pseudopregnant females to produce transgenic offspring. Transgenic animals were identified by Southern blot using tail DNA with a $m$ Wisp3 cDNA probe. Transgenic animals were then mated with 
$129 / \mathrm{SvEv}$ wild-type mice to test for germline transmission of the transgene.

Characterization of mWisp3 transgenic mice Mice were maintained in a specific pathogen free facility and permitted to age with their non-transgenic littermates. Mice were inspected daily for phenotypic findings. Mice were sacrificed and processed for RNA and protein extraction, primary cell culture, and histologic examination at time points ranging from embryonic day 14 (E14) to 62 weeks-of-age.

RT-PCR was performed using total RNA from freshfrozen tissue samples after extraction using Isogen (Nippon Gene Co.) according to the manufacturer's instructions. RT-PCR product was subcloned and sequenced using standard methods.

Histologic specimens were fixed in $10 \%$ buffered formalin (Fisher) before being placed in Tissue-Tek biopsy cassettes (Sakura Finetek). Samples were then processed and embedded in paraffin. Five $\mu \mathrm{m}$-thick tissue sections were collected on Superfrost/Plus slides (Fisher). Images were viewed on a Leica DMLB microscope (Leica Microsystems Inc.) and captured using a Spot Camera and software (Diagnostics Instruments, Inc.).

For Western blotting, entire hearts, kidneys, or femoral articular cartilages were immediately frozen in a methanol/ dry ice bath, homogenized in pestle tubes, and then extracted on ice for $20 \mathrm{~min}$ in $1 \mathrm{~mL}$ Triton X-100 buffer (20 mM Tris-HCl, pH 8.0, $137 \mathrm{mM} \mathrm{NaCl,} \mathrm{1 \%} \mathrm{Triton}$ X-100, 1 mM EGTA, 10\% Glycerol, $1.5 \mathrm{mM} \mathrm{MgCl} 2,1 \mathrm{mM}$ dithiothreitol, $1 \mathrm{mM}$ sodium vanadate, $50 \mathrm{mM}$ sodium fluoride, and proteinase inhibitor mixture (Roche)). The protein concentrations were determined by Bradford Assay, and the samples were stored at $-80^{\circ} \mathrm{C}$ until use.

Primary kidney cell culture Four-week-old $\beta$-actin-WISP3 transgenic and non-transgenic littermates were sacrificed and their kidneys were recovered. After three washes with sterile PBS containing 10\% PSM, the kidneys were minced into small pieces and digested in $10 \mathrm{~mL}$ DMEM/F12 medium containing $1 \mathrm{mg} / \mathrm{mL}$ Collagenase A (Roche) and $10 \% \mathrm{BSA}$ on a $10 \mathrm{~cm}$ dish at $37^{\circ} \mathrm{C}$ for $30 \mathrm{~min}$ in a tissue culture incubator. Following gentle suspension using a pipet, the mixture was passed through a cell strainer into a $50 \mathrm{~mL}$ Falcon tube and centrifuged at 1,000 $\mathrm{g}$ for $10 \mathrm{~min}$. The supernatant was discarded and the cell pellet was suspended in $3 \mathrm{~mL}$ of fresh DMEM/F12 containing 20\% FCS and $1 \%$ PSM. From each transgenic and nontransgenic kidney $1 \times 10^{7}$ cells $/ \mathrm{mL}$ were plated onto $10 \mathrm{~cm}$ tissue culture plates. The medium was changed daily for 2 days. Adherent cells were recovered by trypsin digestion, re-plated, and grown to confluence in another 2 days. Conditioned medium and cell lysate were then recovered, as described above, and stored $-80^{\circ} \mathrm{C}$ until use.
Immunodetection of WISP3 following SDS-PAGE Tissue extract, conditioned medium, and cell lysate samples were electrophoresed in 12\% Tris- $\mathrm{HCl}$ gels (BioRad), transferred to Immobilon $\mathrm{P}$ membrane (Millipore Corp.), and then blocked with $4 \%$ dry milk in Tris-buffered saline (TBS) at $4^{\circ} \mathrm{C}$ overnight. Primary and secondary antibodies were diluted using 4\% dry milk/TBS with $0.05 \%$ Tween 20 (TBST). WISP3-C rabbit polyclonal antibody (Kutz et al. 2005; Nakamura et al. 2007) was used as the primary antibody (1:500 dilution) and goat anti-rabbit HRPconjugated antibody (Pierce) was used as the secondary antibody (1:5,000). ECL plus Western blotting Detection System (Amersham Biosciences) and X-OMAT AR film (Eastman Kodak Company) were used for developing blots and detecting signal.

Generation of expression constructs and in vitro transcription of RNAs for injection into zebrafish embryos Full-length $z$ Wisp3 cloned into the pCS2+ expression vector has been described previously (Nakamura et al. 2007). Full-length mWisp3 was also cloned into the pCS2+ vector. Other cDNA constructs used in this study included human DKK1 ( $h D K K 1)$ and zebrafish Wnt8 (zWnt8) (Nakamura et al. 2007). The RNAs were synthesized from Not I digested pCS2+ plasmids using the sp6 mMessage mMachine kit (Ambion). Phenol red $(0.1 \%)$ was added to the RNA solution as a tracer, and $150 \mathrm{pg}$ of RNA was injected into 1 or 2-cell-stage embryos. Following injections, embryos were cultured in aquatic system water. Phenotypic scoring for evidence of BMP and Wnt inhibition were as previously described (Nakamura et al. 2007).

Co-immunoprecipitation experiments COS7 cells were cultured in DMEM supplemented with $10 \%$ FBS. Cells were plated at $2 \times 10^{5}$ cells per well in six-well culture plates $24 \mathrm{~h}$ prior to transfection. Cells were co-transfected with $2 \mu \mathrm{g}$ of mBMP4-myc plasmid and $0.5 \mu \mathrm{g}$ of mSPC4 plasmid (Nakamura et al. 2007). Twenty-four h after transfection, the culture medium was changed to $1 \mathrm{~mL}$ OPTIMEM (Gibco$\mathrm{BRL}$ ) containing 1\% Knockout SR (Invitrogen). mBMP4myc-containing conditioned medium was collected $48 \mathrm{~h}$ later and stored frozen at $-80^{\circ} \mathrm{C}$ until use. Five-hundred $\mu \mathrm{L}$ of mBMP4-myc-containing conditioned medium was mixed with either $1 \mathrm{~mL}$ of $\beta$-actin-WISP3-transgenic, or nontransgenic, primary kidney cell culture conditioned medium and $65 \mu \mathrm{L}$ of anti-myc beads (Santa Cruz Biotechnology). The samples were rocked overnight at $4^{\circ} \mathrm{C}$. Beads were pelleted, washed four times at $4^{\circ} \mathrm{C}$ for $5 \mathrm{~min}$ each in RIPA buffer (50 mM Tris, pH 8, $150 \mathrm{mM} \mathrm{NaCl}, 1 \% \mathrm{NP}-40,0.5 \%$ deoxycholate, $0.1 \%$ SDS), then resuspended in $65 \mu \mathrm{L} 2 \times$ SDS-PAGE loading buffer with 5\% BME. After boiling for $5 \mathrm{~min}, 20 \mu \mathrm{L}$ were separated by SDS-PAGE, transferred to Immobilon $\mathrm{P}$ and immunodetected using HRP-conjugated 
anti-myc antibody (Santa Cruz Biotechnology) and WISP3-C antibody. Immunoreactive bands were detected as previously described.

Bioassay for BMP signaling in mammalian cells BMP signaling bioassays were performed using mouse $\mathrm{C} 3 \mathrm{H} / 10 \mathrm{~T} 1 / 2$ cells. Cells were plated in 96-well plates $\left(2 \times 10^{4}\right.$ cells/well) and recombinant human BMP2 (rhBMP-2) (R\&D Systems) was added to the culture medium (final concentration $=100 \mathrm{ng} / \mathrm{mL}$ ). Cells were then cultured in $50 \mu \mathrm{L}$ of DMEM $(10 \%$ FBS $)$ and $50 \mu \mathrm{L}$ of $\beta$-actinWISP3-transgenic or non-transgenic primary kidney cell conditioned medium. The culture medium was changed every other day, until the cells were harvested on day 6 . The cells were washed twice with ice-cold PBS and alkaline phosphatase activity was measured with the LabAssay ${ }^{\mathrm{TM}}$ ALP kit (Wako Chemicals USA Inc.) according to the manufacturer's protocol. Total protein content was determined with the BCA Protein Assay kit (Pierce Chemical Co.) using bovine albumin as a standard. Results are expressed as units ALP activity per microgram of total cell protein. Experiments were performed in triplicate on two separate occasions.

\section{Results}

mWisp3 cDNA corresponding to wild-type sequence was subcloned to produce pcDNA3.1 (CMV-WISP3) and pCAGGS ( $\beta$-actin-WISP3) expression vectors, which were then used to transiently transfect HEK293T cells. The constructs produced immunodetectable WISP 3 in the conditioned medium of HEK293T cells (Fig. 1). The constructs were then used to produce transgenic mice.

Sixty-nine and 61 pronuclear injections were performed using the CMV-WISP3 and $\beta$-actin-WISP3 constructs, and $8(12 \%)$ and $10(16 \%)$ transgenic offspring were recovered, respectively. Two lines each for the CMV-WISP3 (lines 9 and 16) and $\beta$-actin-WISP3 (lines 37 and 38) transgenes were maintained. Transgene copy numbers for these lines ranged from 2 to 7 copies as determined by Southern blot analysis (data not shown). Transgenic offspring were obtained at the expected Mendelian ratios, consistent with there being a single insertion site and no embryonic lethality. For the CMV-WISP3 lines, 17 of 38 (line 9) and 6 of 12 (line 16) initial offspring were transgene positive. For $\beta$-actinWISP3 lines 14 of 29 (line 37) and 14 of 27 (line 38) initial offspring were transgene positive. We were surprised by the absence of a phenotype in the transgenic mice and by their recovery at the expected Mendelian frequencies. Therefore, we confirmed that the sequence of the mWisp 3 transgenes in these animals were wild-type by subcloning and sequencing the full-length RT-PCR transcript.
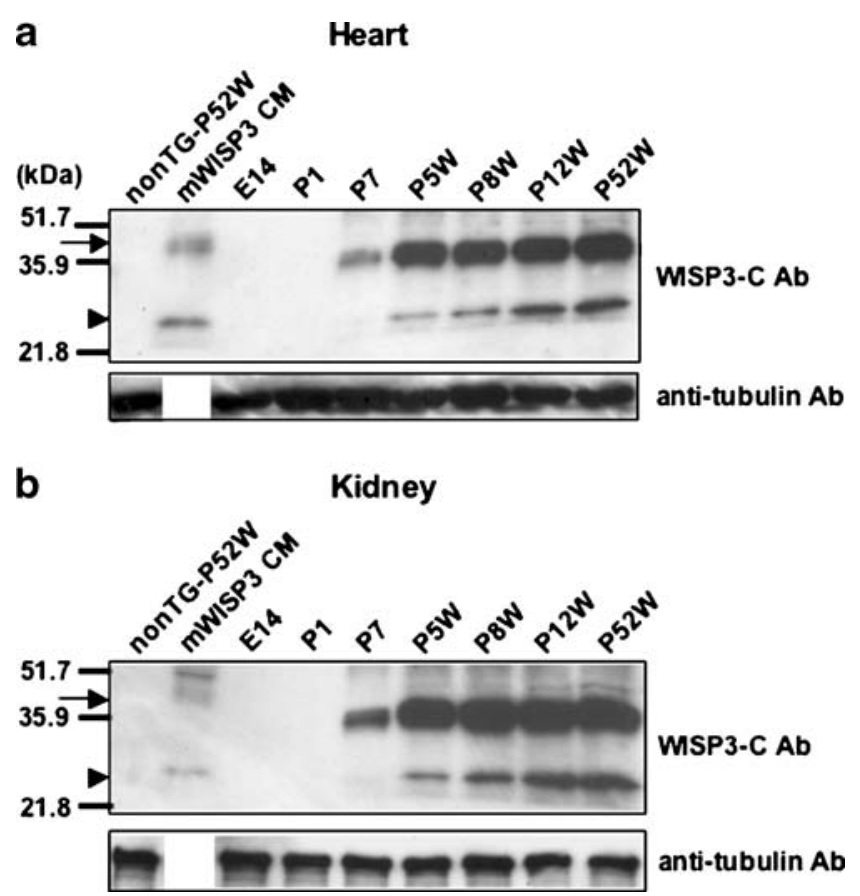

Fig. 1 Detection of mWISP3 in transiently transfected cells and in transgenic mice. a Immunoblots of whole protein extracts of cardiac tissue recovered from $\beta$-actin-WISP3 transgenic mice at embryonic day 14 (E14) and post-natal days 1 (P1) and 7 (P7), and postnatal weeks 5 (P5W), 8 (P8W), 12 (P12W), and 52 (P52W). Extracts were separated by $10 \%$ SDS-PAGE gel and immunodetected using antiWISP3-C antibody. Conditioned medium containing recombinant mWISP3 expressed by HEK293T cells (mWISP3 CM) serves as a positive control (arrow), whereas a cardiac tissue extract from a 52-week-old non-transgenic littermate (nonTG-P52W) serves as a negative control. Note that mWISP3 protein is detected at P7 and at all older ages in the transgenic animals and not in the non-transgenic animal. Also note the presence of a C-terminal WISP3 cleavage product (arrowhead) in the HEK293T conditioned medium and the transgenic cardiac extracts. Immunodetection with an anti-tubulin antibody serves as a loading control. b Immunoblots of whole protein extracts of kidney tissue recovered from $\beta$-actin-WISP3 transgenic mice separated by $10 \%$ SDS-PAGE gel and immunodetected using anti-WISP3-C antibody. Conditioned medium containing recombinant mWISP3 expressed by HEK293T cells (mWISP3 CM) serves as a positive control (arrow) whereas kidney extract from a 52-week-old non-transgenic littermate (nonTG-P52W) serves as a negative control. Note that mWISP3 protein is detected at $\mathrm{P} 7$ and at all older ages in the transgenic animals and not in the non-transgenic animal. Also note the presence of a C-terminal WISP3 cleavage product (arrowhead) in the HEK293T conditioned medium and the transgenic cardiac extracts. Immunodetection with an anti-tubulin antibody serves as a loading control

We performed RT-PCR and detected mWisp3 transgene expression at E14 and P1 in the heart and kidneys of betaactin-WISP3 mice (data not shown), but did not observe immunodetectable WISP3 by Western blot at these time points; however, WISP3 protein was clearly present by P7 and remained readily detectable until at least 52-weeks of age. Failure to detect WISP3 protein prior to $\mathrm{P} 7$ could be due to post-transcriptional regulation of Wisp3 mRNA, which reduces its translation and consequent protein 
expression during embryonic and early postnatal development, or due to requirement for sufficient WISP3 protein to accumulate in tissues before it becomes detectable with the WISP3-C polyclonal antibody. We allowed CMV-WISP3 ( $N=$ $51)$ and $\beta$-actin-WISP3 $(N=62)$ transgenic animals to age along with comparable numbers of non-transgenic littermates $(N=48)$. Visual inspection of the transgenic mice for abnormal behavior, activity, skeletal growth, health, and life expectancy revealed no differences between the transgenics and non-transgenics. The weights of the transgenic mice also did not differ from those of their non-transgenic littermates (male transgenic: $34.33 \pm 0.92 \mathrm{~g}, N=12$; male non-transgenic: $35.28 \pm 1.3 \mathrm{~g}, N=12$ at 9 months old; $p>0.05$ ). Despite clear over-expression of WISP3 in kidney, heart, and cartilage, no histologic differences were observed between the transgenic and non-transgenic mice (Fig. 2a-c).

Because we found no phenotypic differences between transgenic and non-transgenic littermates, but previously found WISP3 to be biologically active when overexpressed in zebrafish and in mammalian cell cultures in vitro, we sought to determine whether WISP3 used in these experiments differed from WISP3 expressed in the transgenic mice. COS7 and $\mathrm{CHO}$ cells are unable to efficiently synthesize and secrete recombinant hWISP3 (Kutz et al., unpublished data), whereas HEK293T cells are able to synthesize and secrete hWISP3 and mWISP3. The apparent molecular weight of HEK293T cell-expressed mWISP3 appears larger than the mWISP3 recovered from transgenic tissue (Fig. 1), but does not substantially differ in size from that recovered from the transgenic primary kidney cell cultures (Fig. 2d). However, we cannot exclude differences in post-translational modifications between WISP3 expressed by HEK293T and expressed by primary kidney cells, since the size of the protein in the conditioned medium is larger than that still undergoing synthesis within the cells (Fig. 2d). Interestingly, the WISP3 C-terminal cleavage fragment observed in medium from HEK293T cells and primary kidney cells (Fig. 2d) was also observed in the transgenic tissue extracts (Fig. 1a, b), indicating that human and mouse cells have the ability to perform this cleavage event. However, we do not yet know whether this cleavage event is biologically significant, since the WISP3 over-expressing mice did not develop a phenotype.

We previously reported that zWISP3 over-expression inhibited BMP and Wnt signaling in zebrafish (Nakamura et al. 2007). The absence of a phenotype in the transgenic mice led us to question whether mWISP3 has this biologic activity. We injected $m$ Wisp3 RNA into fertilized zebrafish oocytes. mWISP3 was able to inhibit BMP signaling in zebrafish, although it was less efficient at doing so than zWISP3 (Fig. 3a). However, unlike zWISP3, which inhibited zWnt8a signaling in zebrafish, mWISP3 had no Wnt inhibitory effect in the zebrafish assay (Fig. 3b). We next studied the in vitro biologic properties of mWISP3 produced by the primary kidney cell cultures from transgenic mice. Co-immunoprecipitation experiments revealed that mWISP3 in primary kidney cell culture conditioned medium could physically interact with mBMP4 (Fig. 3c). To test whether mWISP3 could inhibit BMP signaling we added recombinant human BMP2 (rhBMP2) to mouse $\mathrm{C} 3 \mathrm{H} / 10 \mathrm{~T} 1 / 2$ cells, cultured the cells in primary kidney cell conditioned medium from transgenic, or nontransgenic mice, and looked for a BMP-induced increase in alkaline phosphatase (ALP) activity. Consistent with hWISP3 and zWISP3 having BMP inhibitory activity (Nakamura et al. 2007), conditioned medium from the transgenic kidney cells, but not conditioned medium from the non-transgenic kidney cells, was able to inhibit the BMP2-induced increase in ALP activity (Fig. 3d). These results indicate that $\mathrm{mWISP} 3$ protein expressed by the transgenic mice has biologic activity in this in vitro assay.

\section{Discussion}

We created two strains of transgenic mice that over-express mWisp 3 mRNA and mWISP3 protein driven by the CMV or $\beta$-actin promoters; these promoters drive broad and strong expression of transgenes (Niwa et al. 1991; Yu et al. 2005; Valverde et al. 2008). Surprisingly, mWisp3 transgenic mice were phenotypically indistinguishable from their nontransgenic littermates (Fig. 2). This result was unexpected since we, and other investigators, have identified biologic activities for WISP3 in several different systems (Kleer et al. 2002; Kleer et al. 2004a; Kleer et al. 2004b; Sen et al. 2004; Kutz et al. 2005; Nakamura et al. 2007). Furthermore, we had assumed the low endogenous expression of this protein was an indication of its biologic potency.

In contrast to WISP3, whose deficiency causes the recessive disease PPD in humans and no phenotype in mice, Cyr61-null mice do not survive due to defects in placentation and large blood vessel formation (Mo et al. 2002). CTGF-null mice have a neonatal lethal skeletal dysplasia (Ivkovic et al. 2003) involving endochondral and intramembranous bone growth (Kawaki et al. 2008). Novmutant mice are viable and fertile, but develop cataracts and exhibit skeletal muscle and cardiac problems, including cardiomyopathy and muscle atrophy (Heath et al. 2008). WISP1 and WISP2 mutant mice have not yet been reported; however, to date, no human genetic diseases have been attributed to these genes. Therefore, the precise biologic activities for the $\mathrm{CCN}$ family members have not been clearly defined in vivo, although several activities have been suggested from the in vivo and in vitro experiments (Rachfal and Brigstock 2005; Canalis 2007; Kubota and Takigawa 2007). Despite retaining conserved structural 

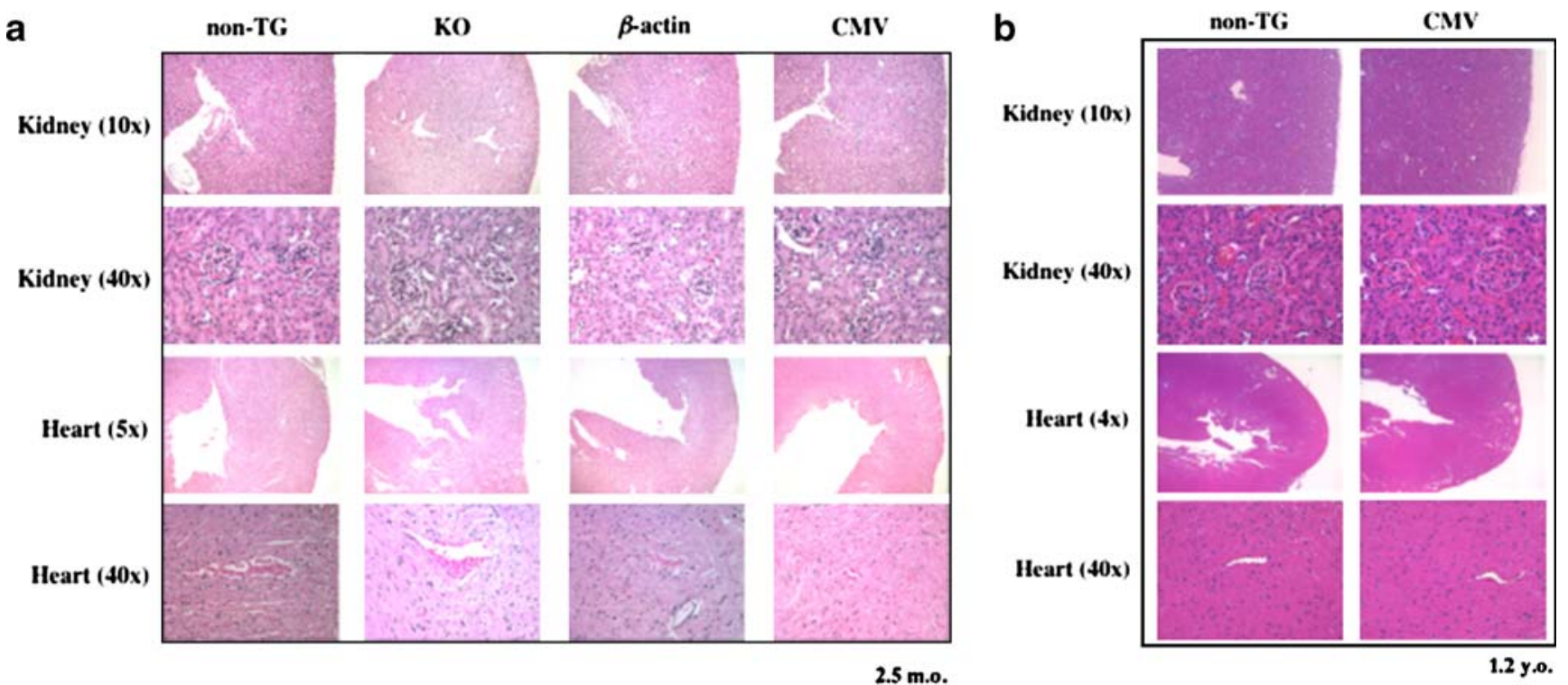

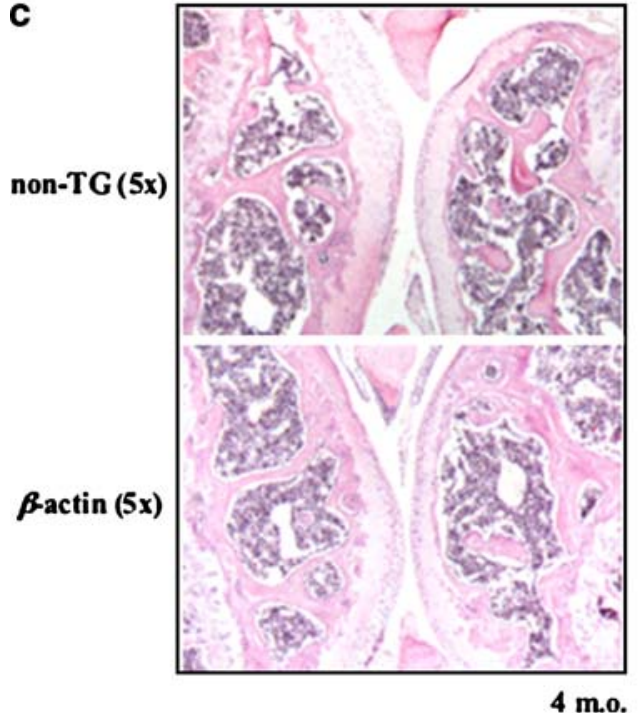

Fig. 2 No histologic difference between Wisp3 knockout, Wisp3 transgenic, and non-transgenic mice. a Photomicrographs of hematoxylin and eosin $(\mathrm{H}$ and $\mathrm{E})$ stained sections of kidneys $(10 \times$ and $40 \times$ magnification) and hearts (5× and 40× magnification) recovered from 2.5-month-old (2.5 m.o.) knockout (KO), CMV-WISP3 transgenic $(\mathrm{CMV})$ and $\beta$-actin-WISP3 transgenic ( $\beta$-actin), and non-transgenic (non-TG) mice revealing no histologic differences between the strains. b Photomicrographs of $\mathrm{H}$ and $\mathrm{E}$ stained sections of kidney and hearts recovered from a 1.2-year-old (1.2 y.o.) CMV-WISP3 transgenic mouse and its non-transgenic littermate. c Photomicrographs of sagittal sections through the femoral-tibial joints recovered from a 4-month-old (4 m.o.)

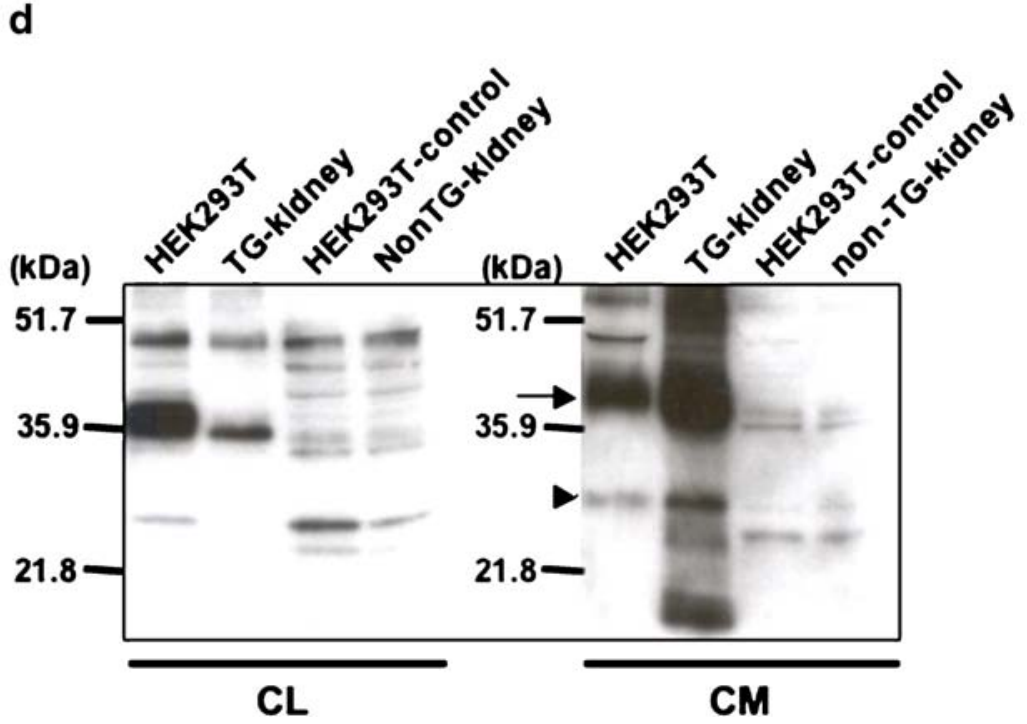

$\beta$-actin-WISP3 mouse and its non-transgenic littermate. $\mathbf{d}$ Immunoblot of mWISP3 in cell lysate (CL) and conditioned medium (CM) from transiently transfected HEK293T cells and primary kidney cell cultures from transgenic mice (TG-kidney) and non-transgenic littermates (nonTG-kidney). Samples were separated by $12 \%$ SDS-PAGE under reducing conditions and probed with the WISP3-C antibody. Intact mWISP3 (arrow) and the WISP3 C-terminal cleavage fragment (arrowhead) are detected. Note that the difference in apparent molecular weight between WISP3 in conditioned medium and in cell lysate is consistent with posttranslational modification. The extent of this modification is similar for mWISP3 expressed by HEK293T cells and by primary kidney cells motifs, the biologic pathways in which these proteins function may have diverged. CTGF has been overexpressed in mice using a Coll1a2 promoter; these transgenic mice developed postnatal onset dwarfism, low bone mass, reduced fertility, and small testis (Nakanishi et al. 2001). Interestingly, the CTGF over-expression phenotype involved tissues in which the Coll1a2 driver was not thought to be active. However, the mechanism by which the phenotypes developed was not explored (Nakanishi et al. 2001). We previously produced mice that over-expressed hWISP3 under the control of Col2al promoter-enhancer; these mice were phenotypically normal (Kutz et al. 2005), prompting us to create the mWisp 3 transgenics that overexpress mWISP3 more broadly, which we describe herein. 

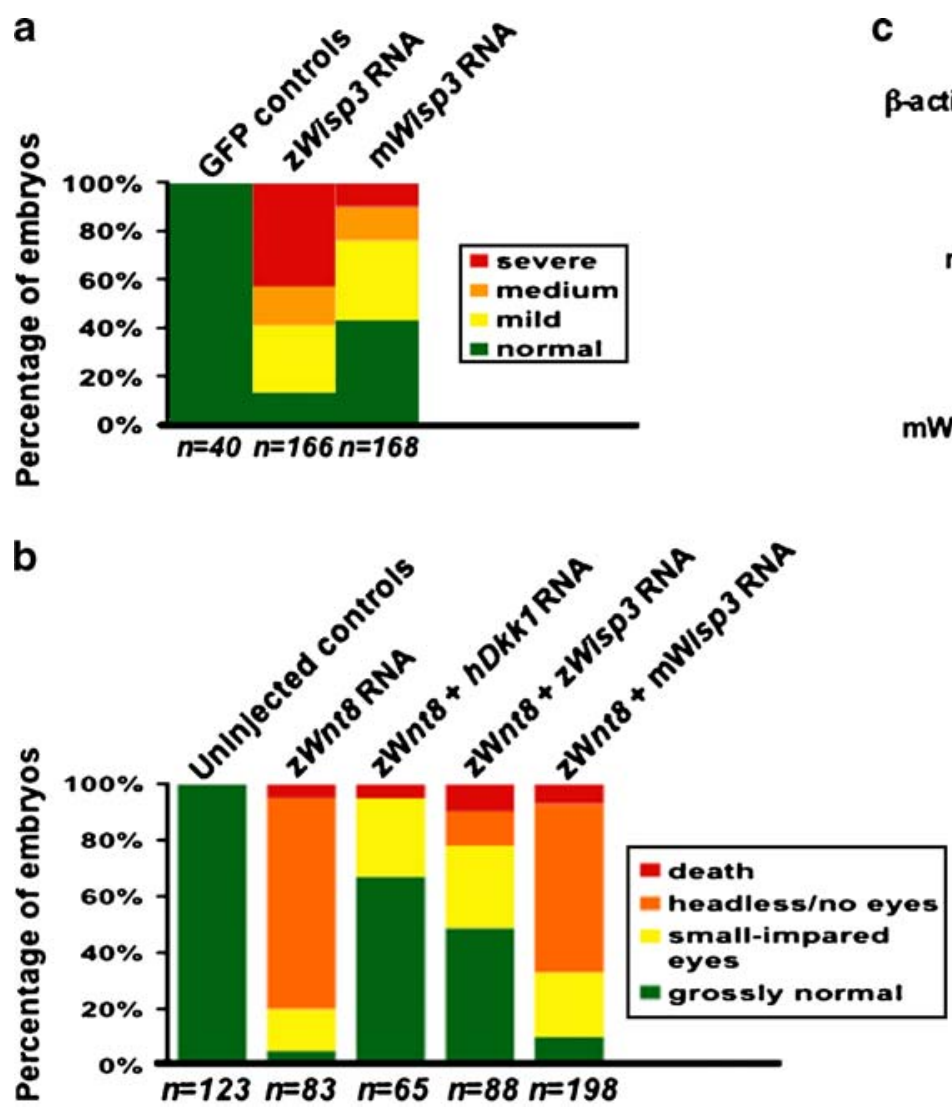
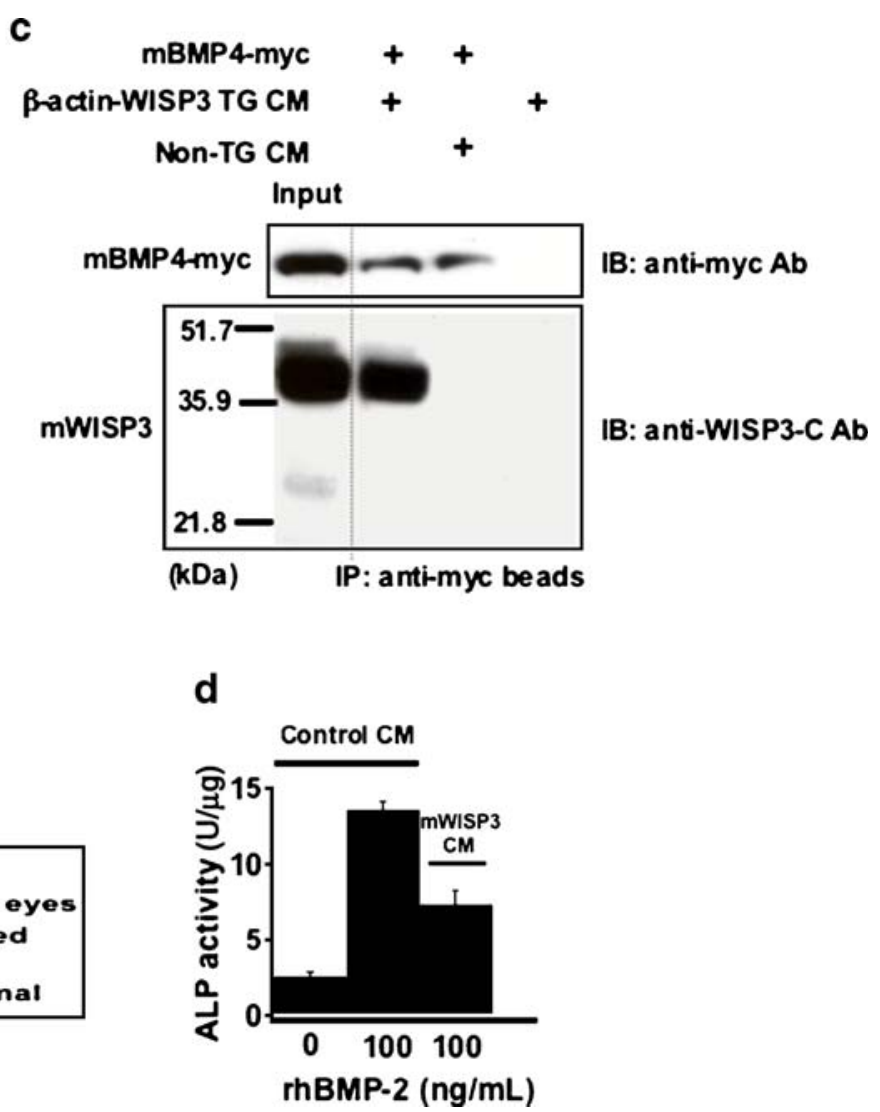

Fig. 3 mWISP3 inhibits BMP signaling in zebrafish embryos and in mammalian cells. a Graph depicting the frequency of the phenotypic outcomes following the injection of zWisp3, mWisp3, or GFP RNA into zebrafish embryos. Embryos classified as "mild" consequences of BMP inhibition correspond to convergence-extension classes, $\mathrm{C} 1$ and $\mathrm{C} 2$, "medium" to $\mathrm{C} 3$ and $\mathrm{C} 4$, and "severe" to $\mathrm{C} 5$ as reported in (Kishimoto et al. 1997). Note that GFP RNA (negative control) had no effect, and that $z$ Wisp 3 was a more potent inhibitor in this assay than $m$ Wisp3. b Graph depicting phenotype frequencies caused by injection of $z W n t 8$ RNA alone, $z$ Wnt8 with $h D K K 1$ RNA, zWisp3 RNA, or mWisp3 RNA. Note that $z W n t 8$ injection causes rostral deformities (death, absent head, small eyes) that are substantially rescued by co-injection of the Wnt inhibitor hDKK1. The zWnt8 phenotype was also inhibited by co-injection of $z$ Wisp 3 , but not by mWisp3. c Immunoblot following co-immunoprecipitation. COS7 cell

We excluded several causes for the lack of a phenotype in the mWISP3 over-expressing mice. We subcloned and sequenced the transgene mRNA transcript and confirmed it had not mutated during transgenesis. We performed Western blots on protein extracts from tissues where the CMV and $\beta$ actin promoters are known to be active and confirmed that immunodetectable mWISP3 was produced (Fig. 1a, b). We injected $m$ Wisp 3 RNA into zebrafish embryos and observed a phenotype consistent with BMP inhibition (Fig. 3a). Another CCN family member, Ctgf, has also been shown to inhibit BMP signaling (Abreu et al. 2002), suggesting that this biologic activity can be conserved within the $\mathrm{CCN}$ family. We also tested mWISP3-containing conditioned conditioned medium containing myc-tagged mBMP4 (mBMP4-myc) was mixed with conditioned medium recovered from primary kidney cell cultures from $\beta$-actin-WISP3 transgenic mice. Following immunoprecipitation with anti-myc antibody, proteins were separated by $12 \%$ SDS-PAGE under reducing conditions and probed with anti-myc antibody (upper panel) and anti-WISP3-C antibody (lower panel). Note that mBMP4 was able to physically interact and co-precipitate intact mWISP3, but not the WISP3 C-terminal cleavage fragment (bottom panel). d Graph depicting the alkaline phosphatase (ALP) activity in $\mathrm{C} 3 \mathrm{H} / 10 \mathrm{~T} 1 / 2$ cells cultured with recombinant human BMP2 (rhBMP2). Note rhBMP2 (100 ng/mL) induces ALP activity and that this induction is reduced when cells were cultured in conditioned medium recovered from primary kidney cell cultures from $\beta$-actinWISP3 transgenic mice compared to conditioned medium recovered from primary kidney cell cultures from non-transgenic mice medium produced by transgenic mouse primary kidney cell cultures and found that the mWISP3 could physically bind BMP and inhibit BMP signaling in mammalian C3H10T1/2 cells (Fig. 3c, d).

The lack of a phenotype in mice over-expressing WISP3 contrasts with the striking phenotypes occurring in transgenic mice that over-express the BMP inhibitor Noggin. Podocytespecific over-expression caused massive mesangial expansion in kidneys (Miyazaki et al. 2006). Osteoblast-specific overexpression of Noggin caused reduced bone mass and skeletal fragility (Devlin et al. 2003). Follciular over-expression of Noggin caused hair loss (Kulessa et al. 2000) and intestinal over-expression caused ectopic villus crypt 
formation (Haramis et al. 2004). We cannot explain why biologic activities for WISP3 can be demonstrated in cultured cells and in zebrafish, but not in live mice. It is possible that the in vivo activity of WISP3 may be tightly regulated by components within tissues that are absent in cell cultures where protein may be released into medium. An alternative possibility is that evolutionary divergence has led to WISP3 becoming non-functional in mice.

Acknowledgements We thank Dr. Junichi Miyazaki for sharing the $\beta$ actin (pCAGGS) vector. M.L.W. is an Investigator with the Howard Hughes Medical Institute. This work was supported by a Clinical Scientist Award in Translational Research from the Burroughs Wellcome Fund and NIH grant T32HD07104.

Open Access This article is distributed under the terms of the Creative Commons Attribution Noncommercial License which permits any noncommercial use, distribution, and reproduction in any medium, provided the original author(s) and source are credited.

\section{References}

Abreu JG, Ketpura NI, Reversade B, De Robertis EM (2002) Connective-tissue growth factor (CTGF) modulates cell signalling by BMP and TGF-beta. Nat Cell Biol 4:599-604

Avsian-Kretchmer O, Hsueh AJ (2004) Comparative genomic analysis of the eight-membered ring cystine knot-containing bone morphogenetic protein antagonists. Mol Endocrinol 18:1-12. doi:10.1210/me.2003-0227

Brigstock DR (2003) The CCN family: a new stimulus package. J Endocrinol 178:169-175. doi:10.1677/joe.0.1780169

Brunkow ME, Gardner JC, Van Ness J, Paeper BW, Kovacevich BR, Proll S, Skonier JE, Zhao L, Sabo PJ, Fu Y, Alisch RS, Gillett L, Colbert T, Tacconi P, Galas D, Hamersma H, Beighton P, Mulligan J (2001) Bone dysplasia sclerosteosis results from loss of the SOST gene product, a novel cystine knot-containing protein. Am J Hum Genet 68:577-589. doi:10.1086/318811

Canalis E (2007) Nephroblastoma overexpressed (Nov) is a novel bone morphogenetic protein antagonist. Ann N Y Acad Sci 1116:50-58. doi:10.1196/annals.1402.055

Chen CC, Lau LF (2008) Functions and mechanisms of action of $\mathrm{CCN}$ matricellular proteins. Int J Biochem Cell Biol

Devlin RD, Du Z, Pereira RC, Kimble RB, Economides AN, Jorgetti V, Canalis E (2003) Skeletal overexpression of noggin results in osteopenia and reduced bone formation. Endocrinology 144:1972-1978. doi:10.1210/en.2002-220918

el-Shanti HE, Omari HZ, Qubain HI (1997) Progressive pseudorheumatoid dysplasia: report of a family and review. J Med Genet 34:559-563

Haramis AP, Begthel H, van den Born M, van Es J, Jonkheer S, Offerhaus GJ, Clevers H (2004) De novo crypt formation and juvenile polyposis on BMP inhibition in mouse intestine. Science 303:1684-1686

Heath E, Tahri D, Andermarcher E, Schofield P, Fleming S, Boulter CA (2008) Abnormal skeletal and cardiac development, cardiomyopathy, muscle atrophy and cataracts in mice with a targeted disruption of the Nov (Ccn3) gene. BMC Dev Biol 8:18

Hurvitz JR, Suwairi WM, Van Hul W, El-Shanti H, Superti-Furga A, Roudier J, Holderbaum D, Pauli RM, Herd JK, Van Hul EV, Rezai-Delui H, Legius E, Le Merrer M, Al-Alami J, Bahabri SA,
Warman ML (1999) Mutations in the CCN gene family member WISP3 cause progressive pseudorheumatoid dysplasia. Nat Genet 23:94-98

Itasaki N, Jones CM, Mercurio S, Rowe A, Domingos PM, Smith JC, Krumlauf R (2003) Wise, a context-dependent activator and inhibitor of Wnt signalling. Development 130:4295-4305

Ivkovic S, Yoon BS, Popoff SN, Safadi FF, Libuda DE, Stephenson RC, Daluiski A, Lyons KM (2003) Connective tissue growth factor coordinates chondrogenesis and angiogenesis during skeletal development. Development 130:2779-2791

Kawaki H, Kubota S, Suzuki A, Lazar N, Yamada T, Matsumura T, Ohgawara T, Maeda T, Perbal B, Lyons KM, Takigawa M (2008) Cooperative regulation of chondrocyte differentiation by $\mathrm{CCN} 2$ and $\mathrm{CCN} 3$ shown by a comprehensive analysis of the $\mathrm{CCN}$ family proteins in cartilage. J Bone Miner Res 23:1751-1764

Kishimoto Y, Lee KH, Zon L, Hammerschmidt M, Schulte-Merker S (1997) The molecular nature of zebrafish swirl: BMP2 function is essential during early dorsoventral patterning. Development 124:4457-4466

Kleer CG, Zhang Y, Pan Q, Gallagher G, Wu M, Wu ZF, Merajver SD (2004a) WISP3 and RhoC guanosine triphosphatase cooperate in the development of inflammatory breast cancer. Breast Cancer Res 6:R110-115

Kleer CG, Zhang Y, Pan Q, Merajver SD (2004b) WISP3 (CCN6) is a secreted tumor-suppressor protein that modulates IGF signaling in inflammatory breast cancer. Neoplasia 6:179-185

Kleer CG, Zhang Y, Pan Q, van Golen KL, Wu ZF, Livant D, Merajver SD (2002) WISP3 is a novel tumor suppressor gene of inflammatory breast cancer. Oncogene 21:3172-3180

Kubota S, Takigawa M (2007) Role of CCN2/CTGF/Hcs24 in bone growth. Int Rev Cytol 257:1-41

Kulessa H, Turk G, Hogan BL (2000) Inhibition of Bmp signaling affects growth and differentiation in the anagen hair follicle. Embo J 19:6664-6674

Kutz WE, Gong Y, Warman ML (2005) WISP3, the gene responsible for the human skeletal disease progressive pseudorheumatoid dysplasia, is not essential for skeletal function in mice. Mol Cell Biol 25:414-421

Leask A, Abraham DJ (2006) All in the CCN family: essential matricellular signaling modulators emerge from the bunker. J Cell Sci 119:4803-4810

Miyazaki Y, Ueda H, Yokoo T, Utsunomiya Y, Kawamura T, Matsusaka T, Ichikawa I, Hosoya T (2006) Inhibition of endogenous BMP in the glomerulus leads to mesangial matrix expansion. Biochem Biophys Res Commun 340:681-688

Mo FE, Muntean AG, Chen CC, Stolz DB, Watkins SC, Lau LF (2002) CYR61 (CCN1) is essential for placental development and vascular integrity. Mol Cell Biol 22:8709-8720

Nakamura Y, Weidinger G, Liang JO, Aquilina-Beck A, Tamai K, Moon RT, Warman ML (2007) The CCN family member Wisp3, mutant in progressive pseudorheumatoid dysplasia, modulates BMP and Wnt signaling. J Clin Invest 117:3075-3086

Nakanishi T, Yamaai T, Asano M, Nawachi K, Suzuki M, Sugimoto T, Takigawa M (2001) Overexpression of connective tissue growth factor/hypertrophic chondrocyte-specific gene product 24 decreases bone density in adult mice and induces dwarfism. Biochem Biophys Res Commun 281:678-681

Niwa H, Yamamura K, Miyazaki J (1991) Efficient selection for highexpression transfectants with a novel eukaryotic vector. Gene 108:193-199

Perbal B (2004) CCN proteins: multifunctional signalling regulators. Lancet 363:62-64

Piccolo S, Agius E, Leyns L, Bhattacharyya S, Grunz H, Bouwmeester T, De Robertis EM (1999) The head inducer Cerberus is a multifunctional antagonist of Nodal, BMP and Wnt signals. Nature 397:707-710 
Rachfal AW, Brigstock DR (2005) Structural and functional properties of CCN proteins. Vitam Horm 70:69-103

Sen M, Cheng YH, Goldring MB, Lotz MK, Carson DA (2004) WISP3-dependent regulation of type II collagen and aggrecan production in chondrocytes. Arthritis Rheum 50:488-497

Sewairi W, Warman ML (2003) Wisp3 and progressive pseudorheumatoid dysplasia. In: Epstein CJ, Wynshaw-Boris A, Erickson RP (eds) Molecular basis of inborn errors of development. Oxford University Press, Oxford, pp 282-283

Spranger J, Albert C, Schilling F, Bartsocas C (1983) Progressive pseudorheumatoid arthropathy of childhood (PPAC): a hereditary disorder simulating juvenile rheumatoid arthritis. Am J Med Genet 14:399-401

Valverde P, Zhang J, Fix A, Zhu J, Ma W, Tu Q, Chen J (2008) Overexpression of bone sialoprotein leads to an uncoupling of bone formation and bone resorption in mice. J Bone Miner Res 23:1775-1788

Wynne-Davies R, Hall C, Ansell BM (1982) Spondylo-epiphysial dysplasia tarda with progressive arthropathy. A "new" disorder of autosomal recessive inheritance. J Bone Joint Surg Br 64: 442-445

Yu K, Chen Z, Wang S, Gorczynski R (2005) Decreased alloreactivity using donor cells from mice expressing a CD200 transgene under control of a tetracycline-inducible promoter. Transplantation 80:394-401

Zhou HD, Bu YH, Peng YQ, Xie H, Wang M, Yuan LQ, Jiang Y, Li D, Wei QY, He YL, Xiao T, Ni JD, Liao EY (2007) Cellular and molecular responses in progressive pseudorheumatoid dysplasia articular cartilage associated with compound heterozygous WISP3 gene mutation. J Mol Med 85:985-996 